\title{
Crecimiento postnatal diferenciado del neurocráneo y del viscerocráneo en equinos domésticos
}

\author{
DIFFERENTIATED POSTNATAL GROWTH OF THE NEUROCRANIUM AND SPLANCHNOCRANIUM \\ IN DOMESTIC EQUINES
Pere M. Parés-Casanovaa ${ }^{1,5}$, Arcesio Salamanca-Carreño ${ }^{2}$, René Crosby-Granados ${ }^{2}$, Nuno Carolino ${ }^{3}$, José V. Leite ${ }^{4}$, Ruy Dantas ${ }^{4}$, Susana Lopes ${ }^{4}$

\section{Resumen}

Se recurrió al estudio por morfometría geométrica de 42 cráneos de equinos de diversas razas domésticas. Se obtuvo una fotografía digital del plano dorsal de cada cráneo, sobre la que se situaron siete hitos craneométricos, que incluían puntos, tanto del neuro como del viscerocráneo. Se comprueba que el proceso ontogénico de los équidos domésticos se ajusta a la regla del CREA («Cranial Evolutionary Allometric»), por la que existe un patrón alométrico común entre taxones de mamíferos, por el cual las especies más pequeñas presentarían caras más cortas y cráneos más anchos que las especies de mayor tamaño. Se acepta una dolicoprosopia correlacionada con la dolicocefalia, o dicho de otro modo, una hipermorfosis facial (puesto que la anchura facial también tiene un comportamiento similar al de la longitud de la cara) paralela al aumento del tamaño de la cabeza para los équidos domésticos.

Palabras clave: dolicocefalia; dolicoprosopia; heterocronía; hipermorfosis

\section{Abstract}

Geometric morphometry of 42 equine skulls from different domestic breeds was used. A digital photograph of the dorsal plane of each skull was obtained, on which were placed seven craniometric landmarks, involving both the neuro and the splanchnocranium. It is verified that the ontogenic process of domestic equids conforms to the CREA rule (Cranial Evolutionary Allometric), by which there is a common allometric pattern among

\footnotetext{
${ }^{1}$ Dept. de Ciència Animal, Universitat de Lleida, Cataluña, España

${ }^{2}$ Facultad de Medicina Veterinaria y Zootecnia, Universidad Cooperativa de Colombia, sede Arauca, Colombia

${ }^{3}$ Instituto Nacional de Investigação Agrária e Veterinária (INIAV), Vale de Santarém, Portugal

${ }^{4}$ Associaçao de Criadores de Equinos de Raça Garrana (ACERG), Vieira do Minho, Portugal

${ }^{5}$ E-mail: peremiquelp@ca.udl.cat
}

Recibido: 4 de enero de 2018

Aceptado para publicación: 20 de junio de 2018 
taxa of mammals, by which the smaller species would have shorter faces and skulls wider than larger species. A dolichoprosopy correlated with dolichocephaly is accepted, or in other words, a facial hypermorphosis (since the facial width also has a behavior similar to that of the length of the face) parallel to the increase in the size of the head for domestic equids.

Key words: dolichocephaly; heterochrony; dolichoprosopy; hypermorphosis

\section{INTRODUCCIÓN}

La dinámica del crecimiento corporal, incluyendo la velocidad del crecimiento y la forma de las curvas de incremento de las dimensiones de cada parte del cuerpo, son características inherentes de cada especie, con un determinado componente de modulación ambiental (Ávila, 2017). El cráneo de los mamíferos presenta una gran disparidad morfológica y se destaca por su heterocronía o cambios en los momentos de desarrollo (Koyabu et al., 2014). Así, la estructura ósea cefálica se compone de huesos que se conectan entre sí alrededor de su periferia por estructuras de tejido blando conocidas como suturas, que son sitios de deposición de hueso nuevo e importantes durante el desarrollo y crecimiento del cráneo.

En el ser humano, durante los primeros años de vida, el volumen del cerebro aumenta rápidamente y el cráneo sufre rápidos cambios morfológicos en tamaño y forma (Bastir y Rosas, 2005). El neurocráneo, en particular, debe ampliarse para proporcionar protección para el cerebro. El neurocráneo en humanos al nacimiento es normalmente el $25 \%$ de su tamaño adulto, $50 \%$ a los 6 meses y el $65 \%$ al año, con un mínimo crecimiento adicional después de 10 años (Libby et al., 2017). La mayoría de las variaciones en la forma son dependientes de las variaciones en el tamaño (Anzelmo et al., 2012).

La morfoestructura craneal debe considerarse no solo desde un punto de vista estático, sino también dinámico, puesto que aparte de conferir un suporte biomecánico, está sometida cambios ontogénicos. A medida que un animal crece onto-genéticamente en tamaño, tiende a cambiar su forma (Slater y Van Valkenburgh, 2009). Entender pues, en el cráneo, el crecimiento y el cambio de conformación del neuro y víscero-cráneo, que tienen desarrollos diferentes (Cerny et al., 2006) es claramente importante. Desde los inicios de la domesticación, la arquitectura craneofacial de los équidos domésticos se ha transformado e ido fijando en las diferentes razas. Esta plasticidad fenotípica puede también haberse debido a adaptaciones locales en función de las condiciones eco-geográficas, no únicamente a la selección artificial. El estudio de las heterocronías en el cráneo equino responde, pues, por lo menos en una parte, a fenómenos naturales.

La hipótesis de este estudio es analizar los patrones de heterocronía a nivel de cráneo en équidos domésticos, centrando el trabajo en ejemplares púberes, a fin de saber hasta qué grado esta heterocronía se prolonga en el periodo posnatal y, en su caso, cómo se manifiesta.

\section{Materiales y Métodos}

Para esta investigación, se recurrió al estudio por morfometría geométrica de 42 cráneos de caballos domésticos de razas diferentes. Los especímenes se encuentran en las colecciones de la Faculty of Technology and Bioresources, Kazakh National Agrarian University en Almatí (Kazakhstan) $(\mathrm{n}=8)$, Universidad Cooperativa de Colombia 
en Arauca (Colombia) (n=4), Faculty of Animal Science and Aquaculture, Agricultural University en Atenas (Grecia) $(\mathrm{n}=7)$, Instituto Nacional de Investigação Agrária e Veterinária en Vale de Santarém (Portugal) (n=1) y Associaçao de Criadores de Equinos de Raça Garrana en Vieira do Minho (Portugal) $(\mathrm{n}=22)$.

Todos los cráneos presentaban al menos el segundo molar superior $\left(\mathrm{M}^{2}\right)$ erupcionado y, por tanto, entre 2 o más años (Sisson et al., 1982), y sin manifestaciones osteológicas patológicas. No se presentó una solicitud de aprobación ética, puesto que se trabajó con cráneos ya recolectados con otros fines de investigación y exposición. Se desconocía el sexo en la mayoría de los especímenes, de modo que se consideraron las alometrías ontogénicas como convergentes, o sea, con tasas de cambio idénticas en ambos sexos.

\section{Obtención de las Imágenes}

Para cada cráneo fue obtenida una imagen digital en su plano dorsal, con una cámara digital Nikon D1500 equipada con teleobjetivo Nikon DX de 18-105 mm, montada en trípode. Cada cráneo fue colocado en el centro del campo óptico, reposando horizontalmente en su cara ventral, con un patrón de $10 \mathrm{~cm}$. Un conjunto de siete hitos (puntos cráneo-métricos), que englobaban tanto el neuro como el víscero-cráneo (Figura 1) fue digitalizado utilizando el programa TpsDig v. 2.16 (Rohlf, 2016). Los tres hitos sagitales permitían la obtención de longitudes y los otros cuatro la obtención de anchuras. Así, a partir de las coordenadas cartesianas se obtuvieron las distancias lineales de los hitos 13 (longitud cefálica), 1-2 (longitud craneal), 2-3 (longitud facial), 4-5 (anchura cefálica) y 6-7 (anchura facial).

\section{Tratamiento Estadístico}

Puesto que las variables lineales obtenidas no presentaban una distribución normal (W Shapiro-Wilk, $p<0.05$ ), se utilizó el

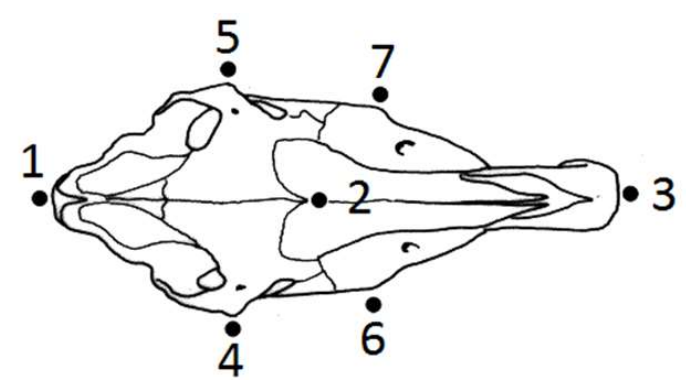

Figura 1. Hitos utilizados (7) para el estudio de la forma en el plano dorsal de los cráneos, que engloban tanto el neuro como el viscerocráneo. A partir de estos hitos se obtuvieron las distancias 1-3 (longitud cefálica), 1-2 (longitud craneal), 2-3 (longitud facial), 4-5 (anchura cefálica) y 6-7 (anchura facial)

test no paramétrico $r_{s}$ de Spearman para el estudio de las correlaciones. Se estableció una regresión lineal multivariante $\gamma$ de todas las variables, utilizando la longitud cefálica como variable independiente, con los datos transformados logarítmicamente. Finalmente, las regresiones de la longitud cefálica con las longitudes craneales y faciales se analizaron con un análisis de covarianza (ANCOVA).

Los análisis se realizaron con el software PAST (Hammer et al., 2001). Se estableció un nivel de confianza del $5 \%$.

\section{Resultados y Discusión}

En el Cuadro 1 se muestran los principales estadísticos descriptivos de las variables en estudio, y en el Cuadro 2 se presentan los coeficientes de correlación $\left(r_{s}\right)$. En todos los casos, las correlaciones fueron significativas $(\mathrm{p}<0.001)$. La regresión multivariante corroboró la significancia $\left(\gamma=0.0001215, F_{4.37}<0.001, \mathrm{p}<0.001\right)$ (Cuadro $3)$. El test ANCOVA reflejó las diferencias en las regresiones con la longitud craneal y facial ( $F=281.4 ; \mathrm{p}<0.001)$, aunque sus distribuciones aparecieron similares $(F=1.393$; $\mathrm{p}=0.241$ ). 
Cuadro 1. Principales estadísticos descriptivos de las variables utilizadas (en $\mathrm{mm}$ )

\begin{tabular}{lccccc}
\hline & $\begin{array}{c}\text { Longitud } \\
\text { cefálica }\end{array}$ & $\begin{array}{c}\text { Longitud } \\
\text { craneal }\end{array}$ & $\begin{array}{c}\text { Longitud } \\
\text { facial }\end{array}$ & $\begin{array}{c}\text { Anchura } \\
\text { facial }\end{array}$ & $\begin{array}{c}\text { Anchura } \\
\text { cefálica }\end{array}$ \\
\hline Valor mínimo & 782.0 & 339.7 & 442.2 & 224.1 & 309.5 \\
Valor máximo & 1293.1 & 602.3 & 691.1 & 359.5 & 499.4 \\
Promedio & 1050.0 & 473.9 & 576.0 & 305.6 & 422.4 \\
Desviación estándar & 120.90 & 63.13 & 69.72 & 32.95 & 46.83 \\
Error estándar & 18.65 & 9.74 & 10.75 & 5.08 & 7.22 \\
Coeficiente de & 11.5 & 13.3 & 12.1 & 10.7 & 11.0 \\
variación (\%) & & & & & \\
\hline
\end{tabular}

Los resultados indican que las relaciones entre las diferentes medidas lineales tienen un comportamiento diferente con el crecimiento (entendido como el aumento de la longitud cefálica). Las longitudes craneal y facial, y la anchura facial mantienen diferente proporción, donde la longitud facial y, en cierta forma, la anchura facial presenta un crecimiento mayor que sus correspondientes craneales. Según la Hipótesis de la Matriz Funcional, que indica que la morfología del cráneo no depende de un control genético propio sino de la influencia de las matrices funcionales asociadas (Anzelmo et al., 2012), se establece la cara como matriz propia, diferente del cráneo. Así, los huesos craneales formarían módulos separados (Bastir y Rosas, 2005), uno explícitamente formado por huesos dermales (lacrimal, parietal, vómer...), y otro, por endocondrales (basisfenoide, exooccipital, exotimpánico, orbioesfenoide, petroso...) (Koyabu et al., 2014). La cara, de origen dermal, podría a su vez ser dividida en cuatro componentes funcionales: óptico, respiratorio, masticatorio y alveolar, pero que por los hitos estudiados (Anzelmo et al., 2012), que están situados en el plano craneal dorsal, no pueden separarse en este estudio. No obstante, puede afirmarse esta prolongación en el tiempo posnatal del crecimiento de los huesos faciales, los dermales.

Los resultados obtenidos corroboran también que la regla del CREA (Cranial Evolutionary Allometric) es aplicable a las especies domésticas de caballos. La regla CREA afirma que existe un patrón alométrico común entre taxones de mamíferos, por el cual las especies más pequeñas presentarían caras más cortas y cráneos más anchos que las especies de mayor tamaño (Linde-Medina, 2016). El comportamiento alométrico en razas sería pues, igual al descrito en especies, ejemplo de una microevolución especial debida a la selección. Se acepta pues una dolicoprosopia (Aparicio, 1960) correlacionada a una dolicocefalia, o dicho de otro modo, una hipermorfosis facial paralela al aumento del tamaño de la cabeza, para los caballares domésticos.

\section{Agradecimientos}

Los autores agradecen a los ganaderos y propietarios, la mayoría anónimos, de las yeguadas de las que se han recolectado cráneos, así como a los gestores de las colecciones osteológicas a las que se permitió nuestro acceso. 
Cuadro 2. Coeficientes de correlación de Spearman $\mathrm{r}_{\mathrm{s}} \mathrm{y}$ valores $p$ entre las variables analizadas $^{1}(\mathrm{n}=42)$

\begin{tabular}{|c|c|c|c|c|c|}
\hline & $\begin{array}{l}\text { Longitud } \\
\text { cefálica }\end{array}$ & $\begin{array}{l}\text { Longitud } \\
\text { craneal }\end{array}$ & $\begin{array}{l}\text { Longitud } \\
\text { facial }\end{array}$ & $\begin{array}{l}\text { Anchura } \\
\text { facial }\end{array}$ & $\begin{array}{l}\text { Anchura } \\
\text { cefálica }\end{array}$ \\
\hline Longitud cefálica & & $6.66 \mathrm{E}-25$ & $4.30 \mathrm{E}-22$ & $1.29 \mathrm{E}-26$ & $6.80 \mathrm{E}-29$ \\
\hline Longitud craneal & 0.965 & & $5.45 \mathrm{E}-14$ & $1.89 \mathrm{E}-20$ & $1.11 \mathrm{E}-22$ \\
\hline Longitud facial & 0.951 & 0.872 & & $4.69 \mathrm{E}-17$ & $8.26 \mathrm{E}-18$ \\
\hline Anchura facial & 0.971 & 0.941 & 0.911 & & $3.37 \mathrm{E}-30$ \\
\hline Anchura cefálica & 0.978 & 0.954 & 0.919 & 0.981 & \\
\hline
\end{tabular}

${ }^{1}$ Coeficientes de correlación en la diagonal inferior y los valores $p$ en la diagonal superior. Todas las correlaciones fueron significativas $(p<0.001)$

Cuadro 3. Exponentes $a$ (inclinación) y $b$ (intersección) para cada variable estudiada en la regresión lineal multivariante ${ }^{1}$, utilizando la longitud cefálica como variable independiente

\begin{tabular}{lcc}
\hline & .a & .b \\
\hline Longitud craneal & 0.961 & -0.220 \\
Longitud facial & 1.031 & -0.365 \\
Anchura facial & 1.021 & -0.607 \\
Anchura cefálica & 0.985 & -0.346 \\
\hline 1 Datos (n=42) obtenidos a partir de la \\
transformación logarítmica con base 10 de \\
las variables
\end{tabular}

\section{Literatura Citada}

1. Anzelmo M, Sardi M, Barbeito A, Pucciarelli H. 2012. Alometrías ontogénicas y dimorfismo sexual facial en dos poblaciones humanas modernas. Rev Argent Antropol Biol 14(1): 89-100.

2. Aparicio G., 1960. Zootecnia especial. Etnología compendiada. $4^{\circ}$ ed. Córdoba: Imp Moderna. 474 p.
3. Ávila D. 2017. Variaciones alométricas durante el crecimiento en siete especies de garzas (Aves: Ardeidae). Rev Biol Trop 65: 1347-1357.

4. Bastir M, Rosas A. 2005. Hierarchical nature of morphological integration and modularity in the human posterior face. Am J Phys Anthropol 128: 26-34. doi: 10.1002/ajpa.20191

5. Cerny R, Horáèek I, Olsson L. 2006. The trabecula cranii: development and homology of an enigmatic vertebrate head structure. Anim Biol 56: 503-518.

6. Hammer Ø, Harper DAT, Ryan PD. 2001. PAST v. $2.17 \mathrm{c}$. Palaeontologia Electronica 4(1): 1-229.

7. Koyabu D, Werneburg I, Morimoto N, Zollikofer C, Forasiepi N, Endo H, Kimura J, et al. 2014. Mammalian skull heterochrony reveals modular evolution and a link between cranial development and brain size. Nature Commun 5: Article 3625. doi: $10.1038 /$ ncomms 4625

8. Libby J, Marghoub A, Johnson D, Khonsari RH, Fagan MJ, Moazen M. 2017. Modelling human skull growth: a validated computational model. J R Soc Interface 14: pii 20170202. doi: 10.1098/ rsif.2017.0202 
9. Linde-Medina, M. 2016. Testing the cranial evolutionary allometric 'rule' in Galliformes. J Evol Biol 29: 1873-1878. doi: $10.1111 /$ jeb. 12918

10. Rohlf FJ. 2016. tpsDig v. 2.26. [Internet]. Available in: http://life.bio.sunysb.edu/ee/rohlf/software.html
11. Sisson S, Grossman JD, Getty R. 1982. Anatomía de los animales domésticos. Barcelona: Salvat. 2302 p.

12. Slater GJ, Van Valkenburgh B. 2009. Allometry and performance: the evolution of skull form and function in felids. J Evol Biol 22: 2278-2287. doi: 10.1111/j.14209101.2009.01845.x 